\title{
Distributed Control of Large Deformable Mirrors
}

\author{
Douglas G. MacMartin ${ }^{a}$, Rikard Heimsten ${ }^{b}$, Torben Andersen ${ }^{b}$ and Mette Owner-Petersen ${ }^{b}$ \\ ${ }^{a}$ Control and Dynamical Systems, California Institute of Technology \\ 1200 E. California Blvd., Pasadena, CA 91125 \\ ${ }^{b}$ Lund Observatory, Lund University Box 43 SE-221 00 Lund, Sweden
}

\begin{abstract}
While it is attractive to integrate a deformable mirror (DM) for adaptive optics (AO) into the telescope itself rather than using relay optics within an instrument, the resulting large DM can be expensive, particularly for extremely large telescopes. A low-cost approach for building a large DM is to use voice-coil actuators, and rely on feedback from mechanical sensors to improve the dynamic response of the mirror sufficiently so that it can be used in a standard AO control system. The use of inexpensive voice-coil actuators results in many lightlydamped structural resonances within the desired control bandwidth. We present a robust control approach for this problem, and demonstrate performance in a closed-loop AO simulation, incorporating realistic models of low-cost actuators and sensors. The first contribution is to demonstrate that high-bandwidth active damping can be robustly implemented even with non-collocated sensors, by relying on the "acoustic limit" of the structure where the modal bandwidth exceeds the modal spacing. Next we introduce a novel local control approach, which significantly improves the high spatial frequency performance relative to collocated position control, but without the robustness challenges associated with a global control approach. The combination of these "inner" control loops results in DM command response that is demonstrated to be sufficient for integration within an AO system.
\end{abstract}

\section{Keywords: Deformable Mirror, Extremely Large Telescopes, Control Systems}

\section{INTRODUCTION AND CONCEPT}

Most modern ground-based optical telescopes use adaptive optics (AO) with one or more deformable mirrors to correct for atmospheric disturbances. It is useful to include large deformable mirrors (DMs) in a telescope to avoid lossy relay optics; these typically have either piezo-electric (displacement) actuators (as in M4 for E-ELT [1]) or voice-coil (force) actuators (as in the LBT M2 [2,3]).

Because displacement actuators effectively constrain the DM at the actuator locations, the structural dynamics of the DM are at much higher frequency than the AO bandwidth. However, while voice-coil actuation has the potential for lower cost, this leads to low frequency dynamics of the mirror structure within the desired AO bandwidth. This presents two challenges that can be overcome with mechanical feedback: significant additional damping is required, and the mirror is much softer (larger response per unit force) at low spatial frequencies than at high. Further, to minimize the cost of implementing the mechanical feedback, it is useful to allow noncollocated sensors (those in $[2,3]$ are collocated) and limited actuator and sensor bandwidth. Here we describe a control strategy to enable a large low-cost DM, and present simulation results within an AO loop; this paper summarizes work first presented in [4-6].

Potential low-cost voice-coil actuators and position sensors have been described in [7] and are shown in Fig. 1. Each actuator is driven by a voice coil, with a linear variable differential transformer (LVDT) for internal feedback (to increase bandwidth). The actuator is connected to the deformable mirror through a suction cup to avoid the need for precision in the physical contact between the mirror and the actuators, and thus reduce cost. Use of commercially available voice coils can further reduce costs, but limit the actuator bandwidth to a few $\mathrm{kHz}$. In contrast, the existing force actuators used for large DMs $[2,3]$ use permanent magnets glued to the back of the mirror, which give a large uniform (flat) frequency response. Low-cost sensors to measure mirror deflection can be made using electret microphones in rubber bellows. However, these sensors do not respond statically, nor is it straightforward to collocate them with the actuators. Thus, a control approach is desired that not only compensates for the mirror flexibility, but accomodates all of these non-ideal characteristics.

The control approach in [2] uses single-input-single-output (SISO) feedback from mirror deflection sensors that are collocated with the actuators. While guaranteed to be robust, feedforward is required to obtain adequate performance at high spatial frequencies, requiring careful calibration. In contrast to this purely collocated approach, Miller and Grocott [8] present a global approach based on a full state-space controller. While overcoming

Adaptive Optics Systems III, edited by Brent L. Ellerbroek, Enrico Marchetti, Jean-Pierre Véran, Proc. of SPIE Vol. 8447, $844710 \cdot$ ○ 2012 SPIE $\cdot$ CCC code: $0277-786 / 12 / \$ 18 \cdot$ doi: $10.1117 / 12.926940$ 


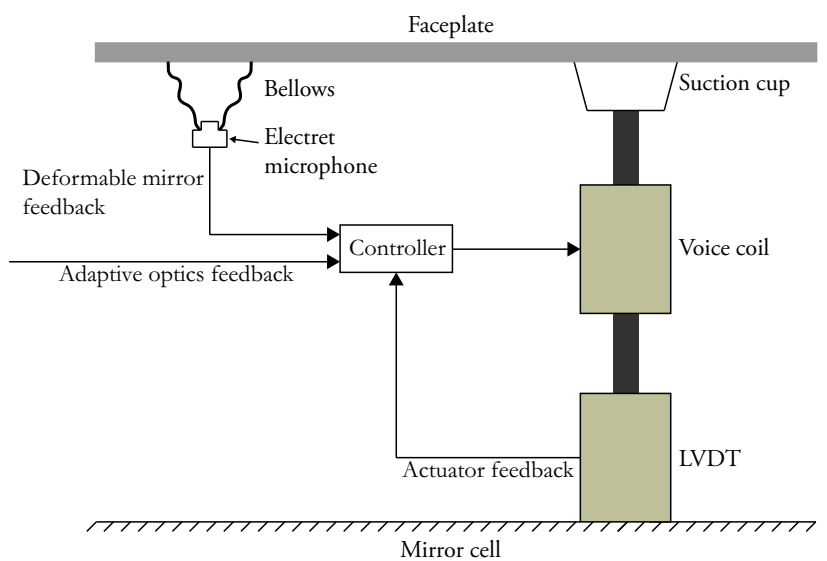

Figure 1. Conceptual design of low-cost force actuators and position sensors. The local and global feedback loops are shown schematically. Cost is reduced by avoiding any need for tight tolerances, and using commercial voice-coils; the finite bandwidth and actuator/sensor non-collocation must be handled by the control approach.

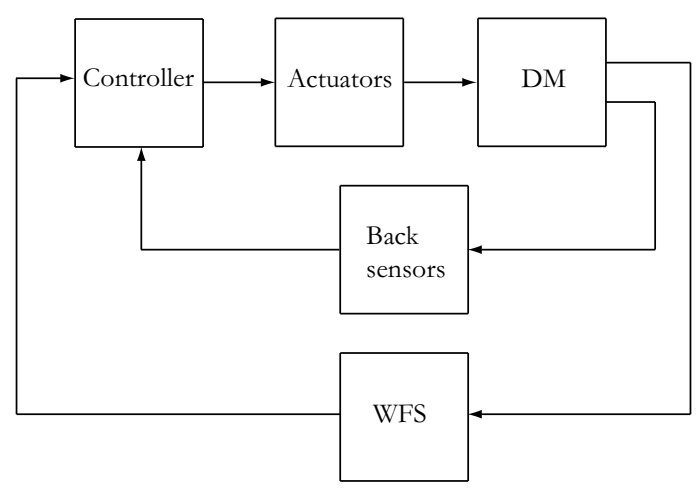

Figure 2. Schematic of overall control architecture, including inner control loop of a flexible, deformable mirror, and outer adaptive optics control loop using wavefront sensor feedback.

the performance limitations of collocated control, this global model-based approach introduces robustness challenges: any control that relies on some particular model information has the potential for insufficient robustness to uncertainty in that information. A distributed control approach has also been simulated for a large deformable mirror [9].

The approach described here (from [4]) is similar in many respects to that in [2]. The approach is divided into two steps: active damping using velocity feedback to compensate for the lightly-damped structural dynamics, and a local (neither collocated nor global) feedforward path to compensate for the difference in static stiffness between low and high spatial frequencies. Motivated by the low-cost sensors in Fig. 1, we first show that active damping can be implemented using non-collocated sensors, and compute an explicit bound on the maximum spatial separation that can be tolerated. We then present a robust local position control strategy that overcomes the high-spatial frequency limitations of collocated position control without introducing the robustness concerns of a model-dependent global feedback strategy; this is similar in spirit to the local estimation strategy in [10]. Finally, we simulate the performance with an outer AO loop (summarizing [6]) in order to verify that the performance is not limited by the remaining differences in response between low and high spatial frequencies or by the finite bandwidth of the mechanical-sensor feedback loop, and furthermore that the sensors do not need to respond statically. We demonstrate the approach using a finite element model of a 1 m diameter mirror, with realistic actuator and sensor characteristics.

\section{SIMULATION EXAMPLE}

For a case study, we use a $2 \mathrm{~mm}$ thick, $1 \mathrm{~m}$ diameter flat faceplate fixed at the inner rim, with material data given in Table 1 . We chose 372 actuators in a square topology with an actuator pitch of $45 \mathrm{~mm}$ as shown in Fig. 3. The 702 sensors are located between each pair of adjacent actuators, so away from the boundaries, each actuator has four neighbouring sensors. In addition to the actuator and sensor nodes, another 4000 nodes are used in the finite element model. The dynamic behavior of the faceplate is described by

$$
\mathbf{M} \frac{\mathrm{d}^{2} \boldsymbol{\xi}}{\mathrm{d} t^{2}}+\mathbf{E} \frac{\mathrm{d} \boldsymbol{\xi}}{\mathrm{d} t}+\mathbf{K} \boldsymbol{\xi}=\mathbf{f}
$$

\begin{tabular}{clc}
\hline Parameter & Definition & Value \\
\hline$E$ & Young's modulus & $63 \times 10^{9} \mathrm{~Pa}$ \\
$\rho$ & density & $2.23 \times 10^{3} \mathrm{~kg} / \mathrm{m}^{3}$ \\
$\nu$ & Poisson ratio & 0.2 \\
$h$ & DM thickness & $2 \mathrm{~mm}$ \\
$D$ & bending stiffness & $E h^{3} / 12\left(1-\nu^{2}\right)$ \\
$r$ & radius of the mirror & $0.5 \mathrm{~m}$ \\
$A$ & DM area & $\pi r^{2}$ \\
$\zeta$ & damping ratio & $1 \%$ \\
\hline
\end{tabular}

Table 1. Nomenclature and parameter values for a $1 \mathrm{~m}$ deformable mirror used for performance studies. 


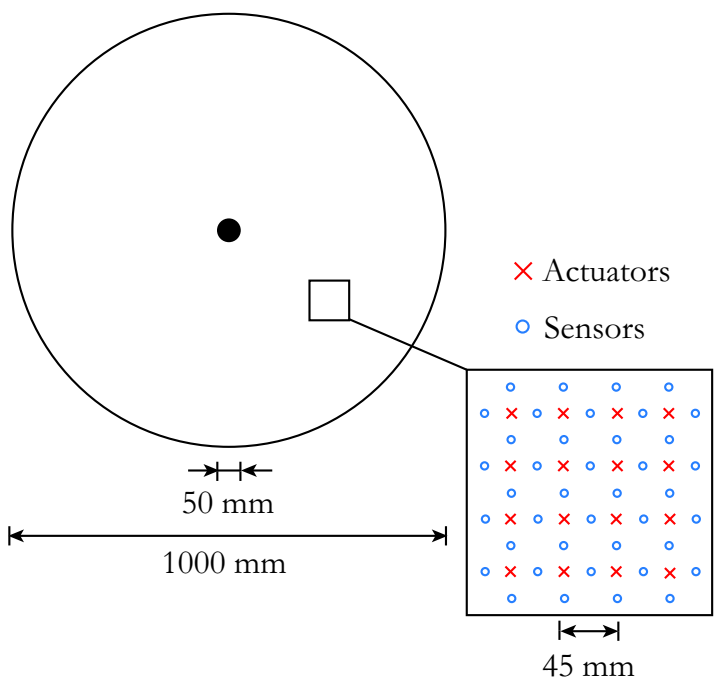

Figure 3. The topology of the actuator and sensor positions for the $1 \mathrm{~m}$ case study.
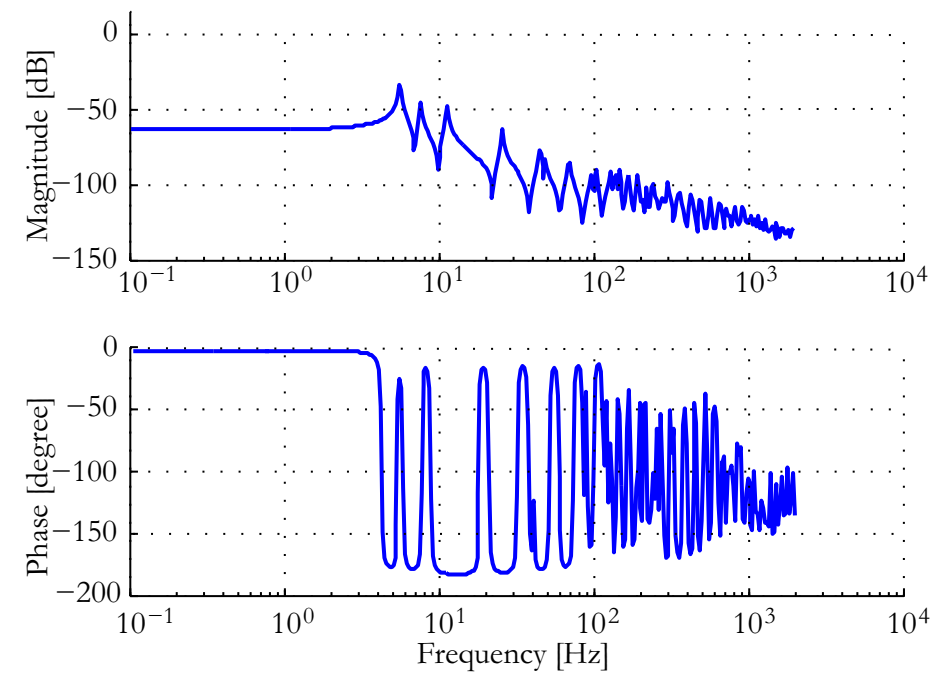

Figure 4. Transfer function of the deformable mirror from force to position (average of four neighboring sensors) at the same location.

where $\mathbf{M}, \mathbf{E}, \mathbf{K}$ are respectively the mass, damping and stiffness matrices, $\mathbf{f}$ is a force vector and $\boldsymbol{\xi}$ is a vector of angular and translational displacements. Guyan reduction and modal truncation [11] were used to obtain a more computationally practical model. Guyan reduction reduced the number degrees of freedom for each node to three, retaining out-of plane translation and the two rotations around the in-plane axes. Modal truncation removed the modes with eigenfrequencies above $10 \mathrm{kHz}$, with the static contribution retained.

The combination of force actuators and a thin mirror leads to a poorly damped system with many structural resonances within the desired bandwidth of the $\mathrm{AO}$ system: The first eigenfrequency is at $5.6 \mathrm{~Hz}$ and there are another 12 eigenfrequencies below $50 \mathrm{~Hz}$, as shown in Fig. 4. Note that results in the next section will instead use a wave-based description of the structural dynamics. Resonances arise due to constructive interference of waves reflecting off system boundaries. At high frequencies, small variations in the material properties, interaction with actuators, or boundary details will result in significant uncertainty in the structural resonances, making any approach that is reliant on exact knowledge of the resonances non-robust. However, the relevant properties of the mirror near an actuator depend only on the local structure properties, and are much less sensitive to modeling errors.

The dynamic behaviour of the actuators and sensors also needs to be included in the model, since both have finite upper bandwidth, and the sensors in Fig. 1 also have non-zero lower bandwidth (they do not respond statically). The suction cup behaves as a spring with stiffness $k$ and damping coefficient $e$, so that the transfer function for an actuator is

$$
\frac{\delta_{\mathrm{r}}}{F}=\frac{1}{\left(m s^{2}+e s+k\right)}
$$

where $m$ is the mass and $\delta_{\mathrm{r}}$ the position of the moving rod, and $F$ is the electromagnetic force developed by the voice coil, which is the product of its force constant and the current in the winding. A local current loop can be added to suppress the influence of the inductance and back electromotive force of the voice coil. The current is then proportional to the input voltage $U$, and the transfer function from voltage to rod displacement (neglecting scaling factors) is

$$
\frac{\delta_{\mathrm{r}}}{U}=\frac{1}{m s^{2}+e s+g+k}
$$

where $g$ is the proportional gain of the local feedback loop from the LVDT.

The sensors on the back of the mirror, shown in Fig. 1, encompass electret microphones inside bellows. An electret microphone can be viewed as a pressure sensor with a flat region of the frequency response between the low-pass cutoff, $\omega_{\mathrm{lp}}$ and the high-pass cutoff, $\omega_{\mathrm{hp}}$. The transfer function (neglecting scaling factors) is

$$
H_{\mathrm{sen}}(s)=\frac{s}{s+\omega_{\mathrm{hp}}} \cdot \frac{\omega_{\mathrm{lp}}}{s+\omega_{\mathrm{lp}}}
$$


For our design, the corner frequencies are $20 \mathrm{~Hz}$ and $5 \mathrm{kHz}$ [7].

The electret microphones can detect a pressure change, $\Delta p$, of around $0.2 \mathrm{~Pa}$. The corresponding volume change is computed from

$$
\Delta v=-\frac{v \Delta p}{1.4 p}
$$

obtained by differentiation of the state equation for an adiabatic ideal gas, where $p$ is the internal pressure in the bellows. Assuming no dynamics in the bellows between the corner frequencies, the volume change is proportional to the deflection of the mirror. With the dimensions chosen, a deflection of about $10 \mathrm{~nm}$ can be sensed.

\section{ACTIVE DAMPING}

It is well known that damping can be added using collocated rate feedback (e.g. [12]):

$$
u_{v}=-K_{v} \dot{y}_{a}
$$

where $y_{a}$ is the deflection at a given location, $K_{v}$ is a gain, and $u_{v}$ is the command to a force actuator collocated with the deflection measurement. The optimal gain is given by the inverse of the dereverberated drive point mobility (the transfer function from force to velocity if the system were completely damped with all "reverberations" removed); this follows from impedance-matching arguments (e.g. [13]). The dereverberated mobility for a plate is the same as the mobility of an infinite plate [14]:

$$
G=\frac{1}{8 \sqrt{D \rho h}}
$$

This feedback is positive real and hence stability is guaranteed. However, because both the dereverberated system dynamics and feedback are constant with frequency, the loop transfer function will be of order one at all frequencies. Phase lag due to actuator-sensor non-collocation, actuator/sensor dynamics, or delays from the electronic implementation will result in any real system not being positive real at some sufficiently high frequency. The system will still be stable if there is sufficient damping (see e.g. [12, Fig. 5.15]); for the DM here, the trade-off between damping and actuator-sensor spacing can be explicitly calculated.

The key observation is that above a certain frequency, the response is no longer dominated by sharp resonance and anti-resonance peaks, because at any given frequency there are multiple modes participating in the response. The transition to this "acoustic" region begins where the half-power bandwidth of any mode exceeds the average modal spacing by a factor of two or three. The half-power bandwidth of each mode is $2 \zeta f_{m}$, where $\zeta$ is the modal damping and $f_{m}$ the undamped eigenfrequency for the mode. For a plate with area $A$, the average modal spacing $[15]$ is:

$$
\Delta f=\frac{2}{A} \sqrt{\frac{D}{\rho h}}
$$

which is roughly $8 \mathrm{~Hz}$ for the simulation parameters here. Thus the transition to acoustic behavior begins around $f_{\mathrm{ac}}=(\Delta f) / \zeta$; with $1 \%$ damping, we should expect to see a smoother transfer function starting at roughly $800 \mathrm{~Hz}$ (c.f. Fig. 4). At sufficiently high frequencies, the phase of the transfer function from force to collocated velocity will be closer to $0^{\circ}$, rather than $\pm 90^{\circ}$; this allows the feedback to roll-off despite phase lag from the implementation. This also implies a minimum rate feedback bandwidth of $f_{\text {ac. }}$. Note that this limit frequency decreases as the deformable mirror increases in size, making larger mirrors easier to control.

Stability can still be guaranteed if the frequency at which positivity breaks down exceeds the acoustic limit. In particular, the phase from non-collocated sensors and actuators is $45^{\circ}$ (and thus perhaps tolerable) when the distance between the actuators and sensors is [4]:

$$
d=\frac{c_{\mathrm{B}}}{8 f_{\mathrm{ac}}}
$$

where the bending wave (group) speed in a flat plate at angular frequency $\omega$ is

$$
c_{\mathrm{B}}=2 \sqrt[4]{\frac{D}{\rho h} \omega^{2}}
$$



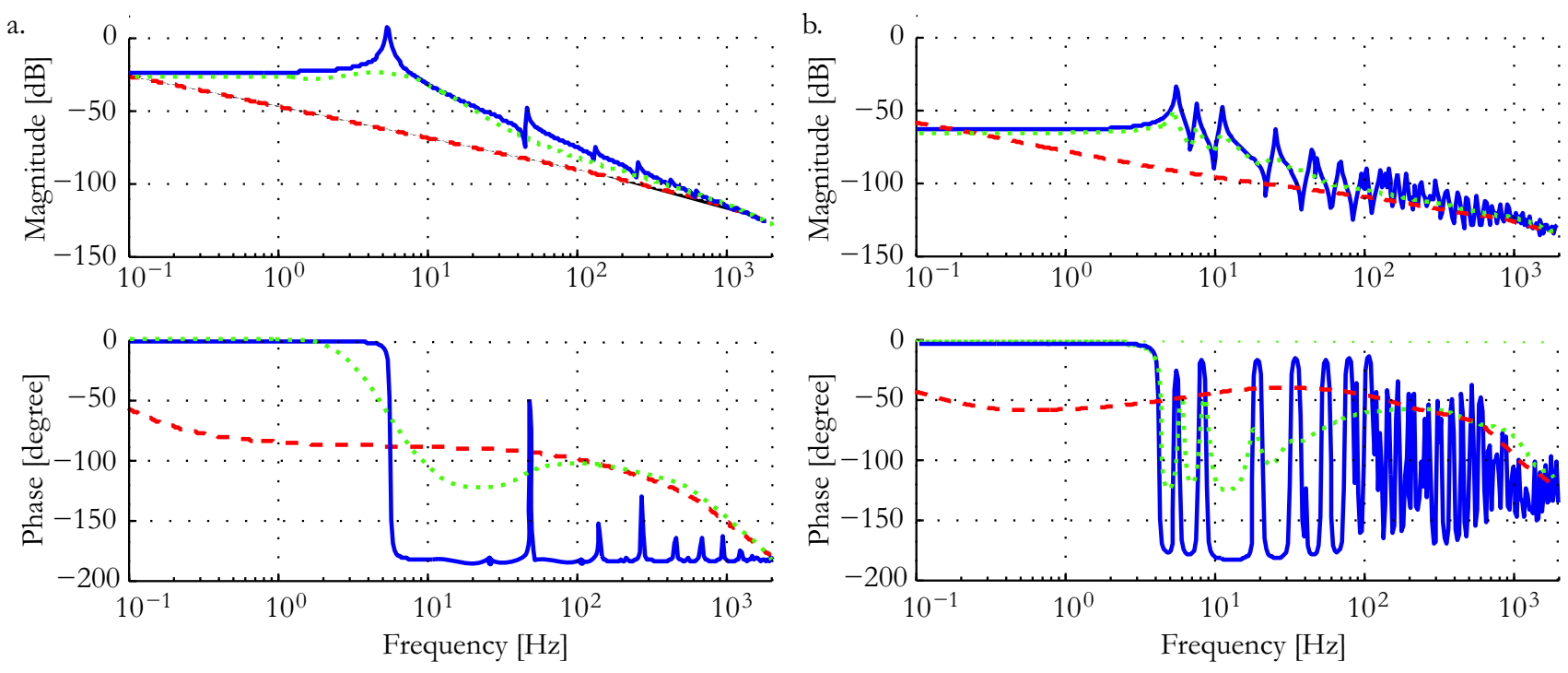

Figure 5. Transfer functions for (a) Global (Case 1), and (b) Local (Case 2). See text for case descriptions. The solid line shows the faceplate dynamics when $\mathbf{K}_{v}$ and $\mathbf{K}_{p}$ are null matrices and $\mathbf{Q}$ in Fig. 6 is an identity matrix. The dashed (red) line illustrates the dynamics with the rate feedback loop closed and ideal sensors, whereas the dotted (green) curve is for non-ideal sensors.

For the properties used in the simulation example, $c_{\mathrm{B}}=\left(3.6 \mathrm{~ms}^{-1 / 2}\right) \sqrt{\omega}$, and the spacing between actuators and sensors should be no more than about $22 \mathrm{~mm}$. (This is the value chosen here; see Fig. 3.) The phase lost to non-collocation is then tolerable at frequency $f_{\text {ac }}$; any additional phase lag due to actuator/sensor bandwidth and implementation delays needs to be accounted for and will reduce the maximum tolerable spacing. Combined with the knowledge that the plant dynamics are relatively smooth above this frequency, which allows roll-off to be introduced, then this demonstrates that robust rate feedback can be used even with non-collocated (and hence inexpensive) sensing. This is a critical enabler for robust control of a distributed force actuated DM.

To demonstrate the properties of the control system, we use the faceplate model introduced earlier as an example, and compute frequency responses for two cases:

- Case 1 (Global): The input command vector $\mathbf{u}$ to all actuators is a pure tip. Its magnitude at a representative location is taken as a scalar input, and the output is the displacement measured at the same location. The objective is to study low spatial frequency behaviour.

- Case 2 (Local): The input command vector, u, has all zeros except at a representative location. The magnitude of the input at that location is the scalar input, and the output is the displacement at the same location. The objective is to study high spatial frequency behaviour.

The frequency response of the mirror with no control is shown as solid curves in Fig. 5 for both Case 1 and 2 . As expected, the first eigenfrequency at $5.6 \mathrm{~Hz}$ corresponds to the tip/tilt mode and is seen both for Case 1 and 2. Closing the SISO rate feedback loops at each actuator with ideal sensors gives the dashed lines in Fig. 5. The gain that maximizes damping at each location makes the system over-damped at low frequencies. With non-ideal sensors (dotted curves), there is hardly any over-damping, since their response drops off below $20 \mathrm{~Hz}$.

\section{POSITION CONTROL}

The ability to robustly implement active damping despite sensor-actuator non-collocation overcomes one of the challenges in using voice-coil actuation, that of lightly damped resonances within the desired control bandwidth. A second issue is that the DM is much more compliant at low spatial frequencies than at high; this is evident from the magnitude intercepts in the transfer functions in Fig. 5. The loop gain of the faceplate is about 30 times higher for low spatial frequencies than for high. As a result, a force command to one actuator produces a global response across the mirror, as shown in Fig. 7(a) for a particular actuator location. To overcome this, an additional matrix $\mathbf{Q}$ is used to improve conditioning, as shown in Fig. 6. 
If $\mathbf{Q}$ were the inverse of the static compliance matrix $\mathbf{C}$ relating motion at the actuator locations to actuator forces (as in [2]), then at least statically, the DM would respond perfectly to commands $u$ at any spatial frequency. However, this requires a global matrix $\mathbf{Q}$ where each actuator command depends on the desired displacement at every other actuator location. Since this relies on global model knowledge, it can be sensitive to small model errors. We instead propose a local scheme that does not rely on knowledge of the entire plant, and therefore has the potential to be more robust [4]. This uses a set of actuator families, each centered on an actuator. A desired displacement command (input to $\mathbf{Q}$ in Fig.6) at a particular actuator results in a force distribution applied to all the actuators in that family.

Given a particular error pattern $\boldsymbol{\xi}$, with $\boldsymbol{\xi}_{k}=1$ at the $k^{\text {th }}$ actuator and $\boldsymbol{\xi}_{j}=0, j \neq k$, then rather than only applying a force to the $k^{\text {th }}$ actuator (which will produce non-zero response across the entire mirror, see Fig. 7(a)), we wish to apply a force distribution that yields a response pattern to counteract the error, see Fig. 7(c). However, we use only actuators close to actuator $k$ (as in Fig. 7(b)), and will tolerate some error in replicating the error pattern.

The force distribution in each family is thus determined by minimizing the cost function

$$
\mathbf{J}=\left\|\mathbf{C f}_{k}-\boldsymbol{\xi}\right\|_{2}
$$

subject to the constraint that elements of the vector $\mathbf{f}_{k}$ not in some set $\Omega_{k}$ must be zero. The set $\Omega_{k}$ can be constructed by including all actuators within some specified distance of $k$. The constrained leastsquares problem is equivalent to solving an unconstrained problem with a truncated matrix $\mathbf{C}_{:, \Omega_{k}}$, indicating that only the columns associated with actuators in $\Omega_{k}$ are retained. Using this approach, the computed force vectors for each family (from the pseudo-inverse of the truncated compliance matrix) are stored as columns in the family matrix $\mathbf{Q}$. Each column corresponds to a command vector for a specific center actuator. This results in a matrix $\mathbf{Q}$ that is an approximate inverse to the compliance C, but using only local information. (Indeed, using the local set of actuators described below, the condition number of $\mathbf{C Q}$ is reduced from roughly $10^{5}$ with $\mathbf{Q}=\mathbf{I}$ to roughly 2, a remarkable improvement using at most $6 \%$ of the actuators to construct each column of the approximate inverse.)

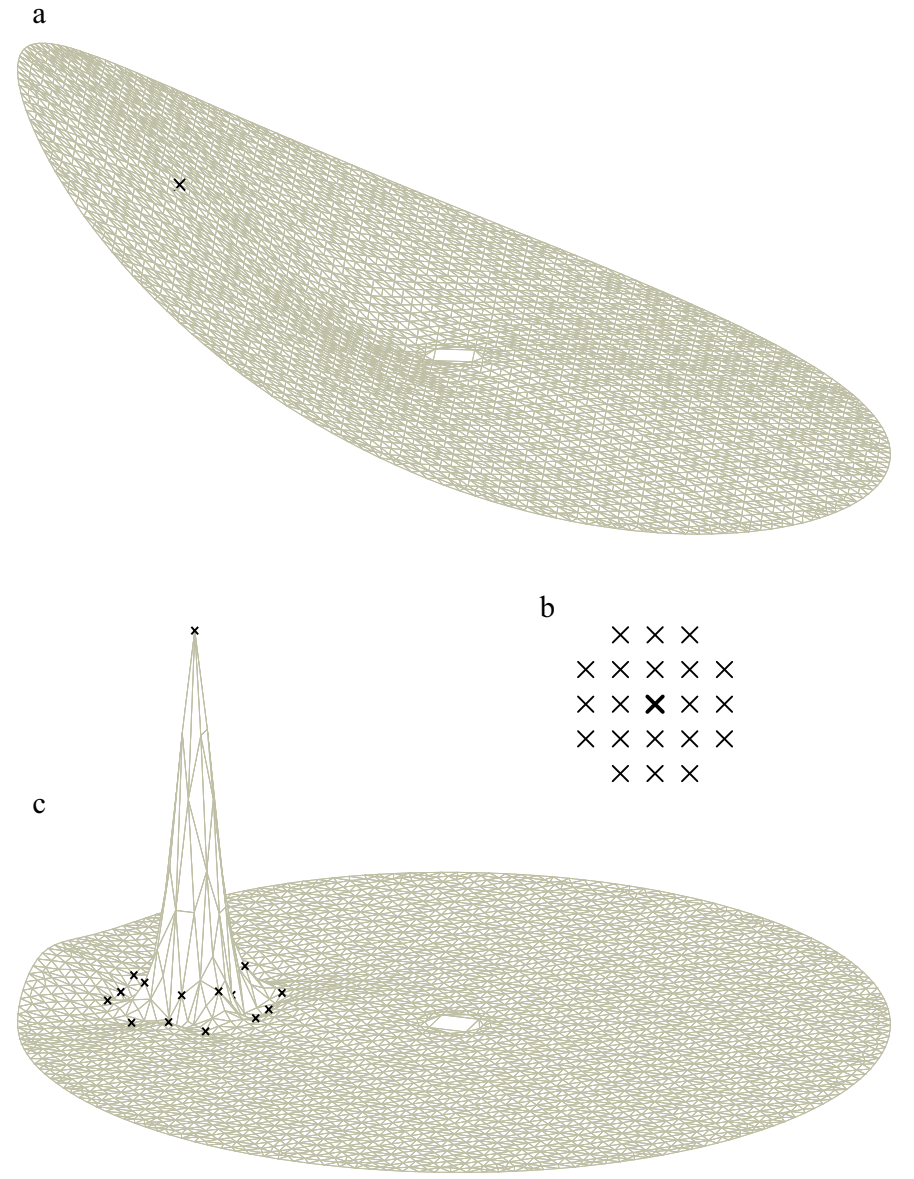

Figure 7. Overview of the performance of the local approach, (a) mirror shape due to a single actuator command $(\mathbf{Q}=\mathbf{I})$, (b) local family group of 21 actuators, (c) mirror shape resulting from unit command to a local actuator family. 


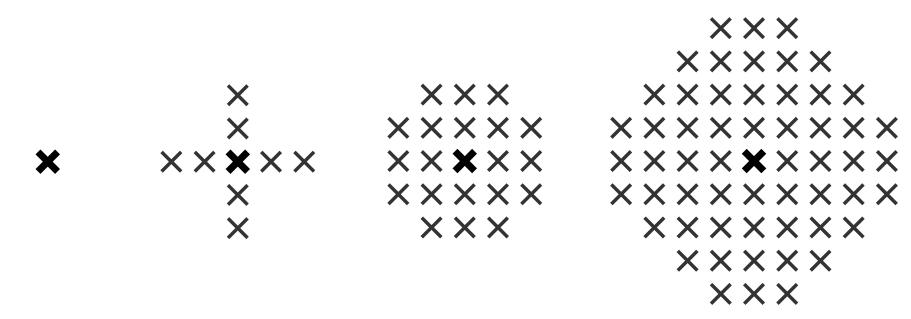

Figure 8. Four family patterns used in [5] to evaluate modal suppression.

To illustrate the effectiveness of this approach, we use the set $\Omega$ illustrated in Fig. 7 (b). The first "ring" of actuators around the center will push in the opposite direction of the center actuator, to counteract the response that would otherwise occur. The second ring of actuators is useful to minimize energy propagation away from the local region, which would otherwise dominate the least-squares performance metric. There is clearly significant potential for exploring different possible sizes for the set $\Omega$; better performance will occur with larger sets, at the expense of robustness due to the requirement for additional structural dynamics information. The appropriate trade-off for a given mirror and actuator/sensor layout can be obtained by looking at the residual least-squares performance metric from (6) as a function of the size and pattern $\Omega$, and choosing the set beyond which there is diminishing benefit from using additional actuators. The four family patterns shown in Fig. 8 were explored in [5], comparing the degree to which low frequency modal excitation is suppressed in the input-output behaviour; simulations in this and the next section illustrate that the set chosen here is sufficient.

Now return to the simulation example, and the low- and high-spatial frequency cases shown in Fig. 5. Including the family matrix $\mathbf{Q}$ in the control system has two related effects. The gain is more similar between low and high spatial frequency modes (equivalently, there is less cross-talk between neighbouring actuators). And because the low spatial frequencies are less easily excited, eigenmodes with eigenfrequencies below $30 \mathrm{~Hz}$ are much less excited by input commands when $\mathbf{Q}$ is included. This is important since these modes are not as well
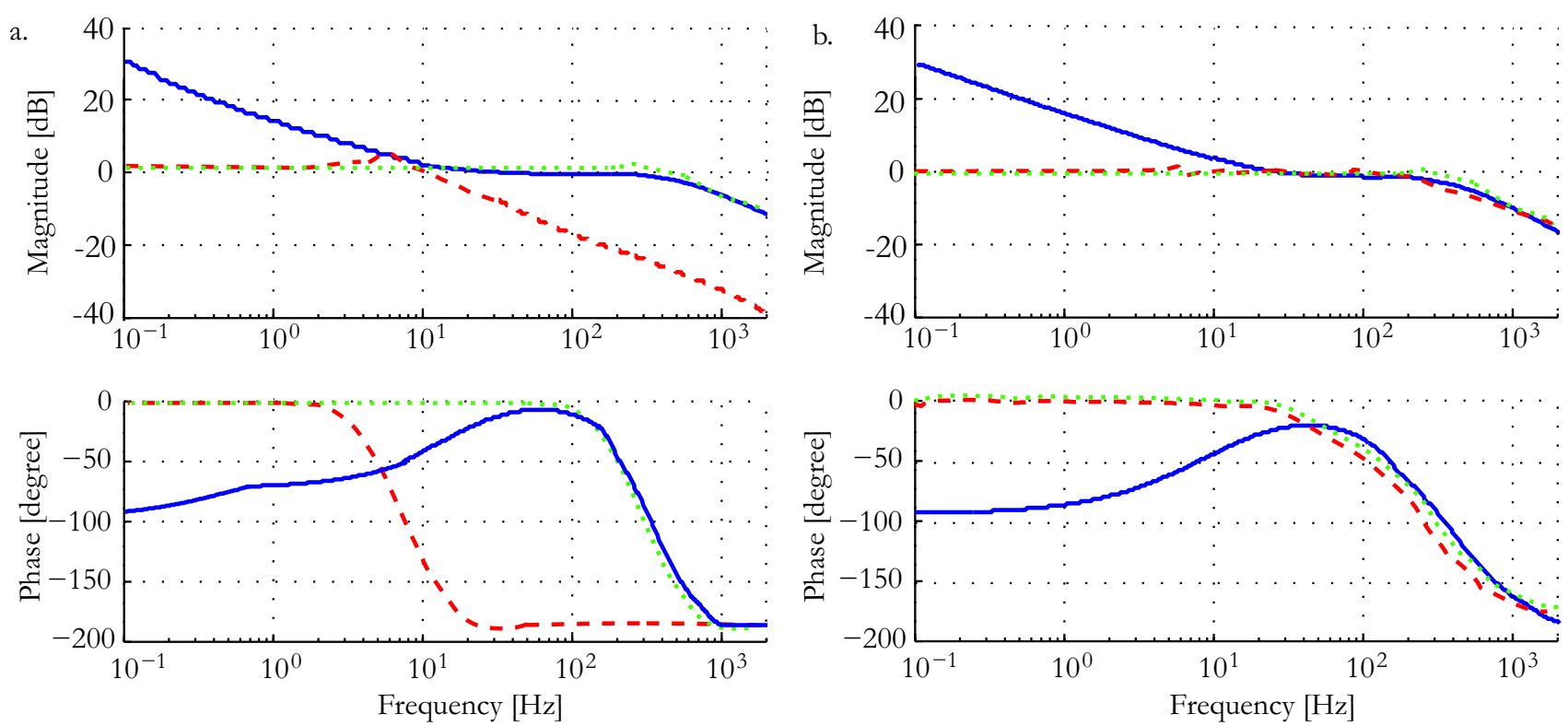

Figure 9. Frequency responses for (a) Case 1 (global), and (b) Case 2 (local). The dashed curves show the effect of the family matrix $\mathbf{Q}$ with non-zero $\mathbf{K}_{v}$ but $\mathbf{K}_{p}=0$; for clarity of illustrating the static response, these are shown with ideal sensors. The magnitude difference (seen in Fig. 5) is no longer present. The dotted curves show the dynamical behavior of the faceplate when the position feedback is also closed, again with ideal sensors. The low-order eigenmodes are shifted towards higher frequencies and the dynamical behavior is similar for the two cases. Finally, the solid curves show the same frequency responses when non-ideal sensors are used. The controlled deformable mirror has similar dynamic behavior for different spatial frequencies. 
damped when using sensors that do not respond quasi-statically.

We continue by looking at the features of the position feedback and the family matrix, with the starting point from the system with rate feedback loops closed (dotted curves in Fig. 5). The magnitude difference of about $30 \mathrm{~dB}$ between the cases is suppressed by the family matrix, shown as the dashed curves in Fig. 9. Also, the low-frequency eigenmodes still present in the high spatial frequency case are attenuated. However, while the low and high spatial frequencies now have similar static gain, the low spatial frequency resonances are below the desired AO bandwidth. This is corrected by adding stiffness through the position feedback $\mathbf{K}_{p}$. To illustrate the effect of the position feedback clearly, the dotted curves in Fig. 9 show the closed-loop performance with ideal sensors. The dynamic behavior is now similar for both cases. Finally, the solid curves include the effect of the non-ideal sensors. The roll off behaviour of the sensors below $20 \mathrm{~Hz}$ gives rise to the observed low-frequency asymptotes in the closed-loop response. The solid curves thus show the dynamical behavior of the deformable mirror system, the next step is to simulate the "outer" AO loop that uses wavefront sensors.

One final detail is that piston is unobservable by the wavefront sensor in an adaptive optics system, and some modification is needed to alleviate unnecessary stress that can slowly build up at the fixed inner rim of the deformable mirror. Rather than driving the piston to zero, a better approach to minimize stresses is to subtract the mean force of the actuators closest to the inner rim from the displacement command at every location with some small gain; this additional feedback loop is shown in Fig. 6.

\section{ADAPTIVE OPTICS CONTROL}

With the control architecture for the inner loop of the deformable mirror described above, we now introduce the outer adaptive optics loop, including wavefront sensing. The dynamic response of the deformable mirror system, shown as the solid lines in Fig. 9, is not the same as that of a typical deformable mirror, but we demonstrate that it is sufficient for the purpose. The feedback control used for the adaptive optics system is shown in Fig. 10, where the plant is the deformable mirror system with the closed position and rate feedback loops as described above and shown in Fig. 6, and

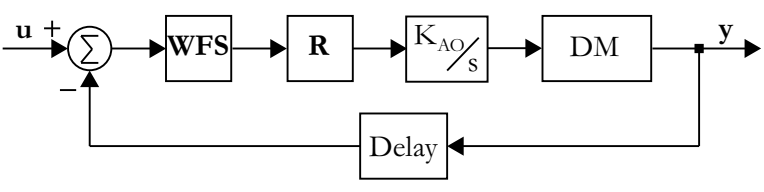

Figure 10. Block diagram of the adaptive optics loop, including wavefront sensor, reconstructor and delay from sampling and computation. The loop is closed with an integral controller indicated by $\mathbf{K}_{\mathrm{AO}} / s$. the input is the commanded displacement.

The WFS-block in Fig. 10 is a matrix representing a ShackHartmann wavefront sensor with a square $22 \times 22$ lenslet array. The lenslet array is aligned such that the corners of the grid coincide with the actuator locations, see Fig. 11. Since the lenslet array map is larger than the deformable mirror, a mask is used to remove 88 subapertures. The four subapertures in the center are omitted since a pure piston displacement of the DM would give a non-zero reading for these due to the inner boundary conditions. The tip and tilt for each remaining subaperture are computed from the wavefront samples at the actuator locations [16]

$$
\begin{aligned}
t i p & =\frac{w\left(\mathbf{p}_{1}\right)+w\left(\mathbf{p}_{2}\right)-w\left(\mathbf{p}_{3}\right)-w\left(\mathbf{p}_{4}\right)}{2} \\
t i l t & =\frac{w\left(\mathbf{p}_{1}\right)+w\left(\mathbf{p}_{3}\right)-w\left(\mathbf{p}_{2}\right)-w\left(\mathbf{p}_{4}\right)}{2}
\end{aligned}
$$

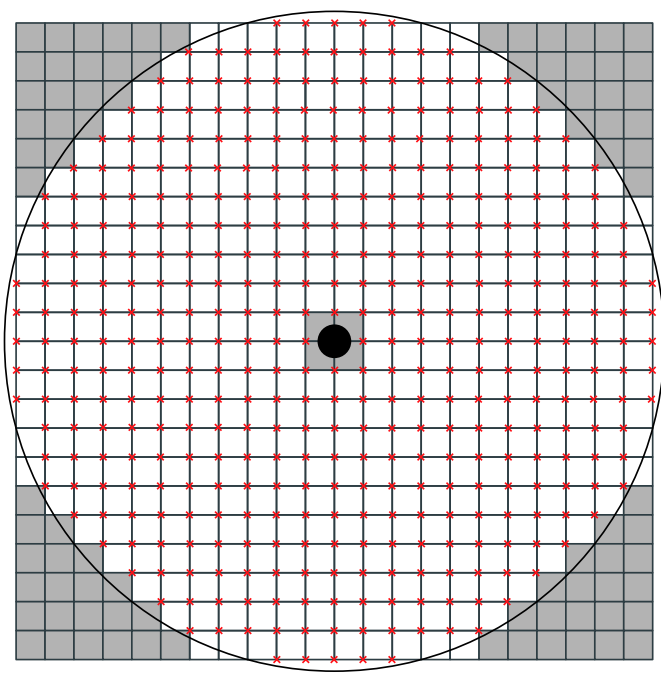

where the two-dimensional vectors $\mathbf{p}_{1}, \mathbf{p}_{2}, \mathbf{p}_{3}$ and $\mathbf{p}_{4}$ define the locations of the four corner points of a subaperture and $w(\mathbf{p})$ is the displacement.

The R-block in Fig. 10 is the reconstructor matrix, used to compute DM displacement commands from the tip and tilt WFS readings. It is assembled using singular value decomposition of the interaction matrix (the matrix that describes the subaperture

Figure 11. The lenslet array grid over the deformable mirror. Lenslet which are not completely filled by the light beam, are not taken into account. Actuator locations are marked by crosses. wavefront tip/tilt when poking each DM displacement individually). 

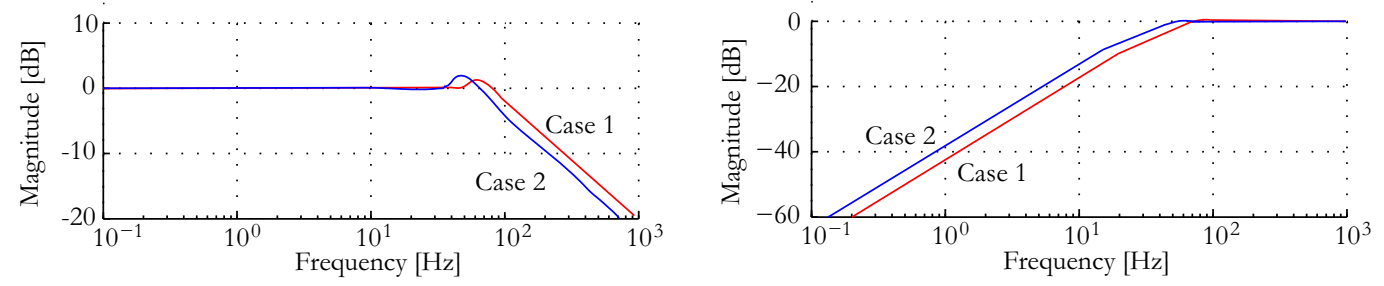

Figure 12. The closed-loop tracking response (left) and rejection (right) of the system shown in Fig. 10. The two cases represent the lowest and highest spatial frequencies that the deformable mirror can achieve.

The performance of the adaptive optics loop is limited by the phase lag introduced by wavefront sampling $(T / 2)$ and zero-order-hold $(T / 2)$ and represented by the delay block in the control loop in Fig. 10 . The delay is modeled as a fifth order Padé approximation of $e^{-s T}$. The closed-loop tracking and rejection performance are shown in Fig. 12 for the same two cases described earlier, with $T=1 \mathrm{~ms}$. The two cases represent the lowest and highest spatial frequencies that the deformable mirror can achieve. An ideal DM would have uniform behaviour for all spatial frequencies, and a flat tracking response up to much higher frequency than the desired AO bandwidth. The differences between the dynamics of our deformable mirror system and an ideal DM include the small difference in behaviour between the different spatial frequencies, an increase in low-frequency gain and some additional phase lag at higher frequencies (see Fig. 9). The latter would not become a significant factor unless the desired AO bandwidth was higher than $100 \mathrm{~Hz}$.

To simulate our DM with the AO loop closed and verify that the non-ideal aspects do not limit AO performance, we assume that the deformable mirror is the secondary in a two-mirror, $30 \mathrm{~m}$ telescope with adaptive optics, with the observing wavelength taken to be $2200 \mathrm{~nm}$. A three layer atmosphere model is used with parameters taken from a study of the atmosphere over the La Palma observatory as given in Table 2. The altitude of the atmospheric layers are not of importance since only objects at zenith are considered and the field is small. There are 20 actuators across the deformable mirror with an actuator pitch of $45 \mathrm{~mm}$, thus the actuator pitch matches the Fried parameter of the first atmospheric layer.

A thin-layer model of the atmosphere is used with nearfield propagation. Assuming Kolmogorov turbulence, the twodimensional power spectrum of the phase is given by [16]:

$$
P_{\text {atm }}(\vec{f})=\frac{0.0229}{r_{0}^{5 / 3} f^{11 / 3}}
$$

where $r_{0}$ is the Fried parameter and $f$ is the magnitude of the spatial frequency vector $\vec{f}$.

We now evaluate the $\mathrm{AO}$ performance in both the temporal and the frequency domain.

\subsection{Performance for Different Zernike Polynomials}

It is useful to expand the atmospheric phase into series of Zernike polynomials. The power spectra for the individual Zernike polynomials, $P(\vec{f})$, can be computed from the power spectrum of the atmospheric turbulence as $[17]$

$$
P(\vec{f})=|M(\vec{f})|^{2} P_{\text {atm }}(\vec{f})
$$

where $M(\vec{f})$ is the Fourier transform of a specific Zernike polynomial. The Fourier transform of the Zernike polynomials is given by $[18]$

$$
|M(\vec{f})|=\sqrt{n+1} \frac{2\left|J_{n+1}\left(\pi D_{\mathrm{DM}} f\right)\right|}{\pi D_{\mathrm{DM}} f} \times \begin{cases}\sqrt{2}|\cos (m \theta)| & \text { for } m \neq 0 \\ \sqrt{2}|\sin (m \theta)| & \text { for } m \neq 0 \\ 1 & m=0\end{cases}
$$

where $n$ is the radial degree, $m$ the the azimuthal frequency of the polynomial, $D_{\text {DM }}$ diameter of the deformable mirror and $J_{k}(x)$ is the Bessel function of the first kind of the order $k$. 

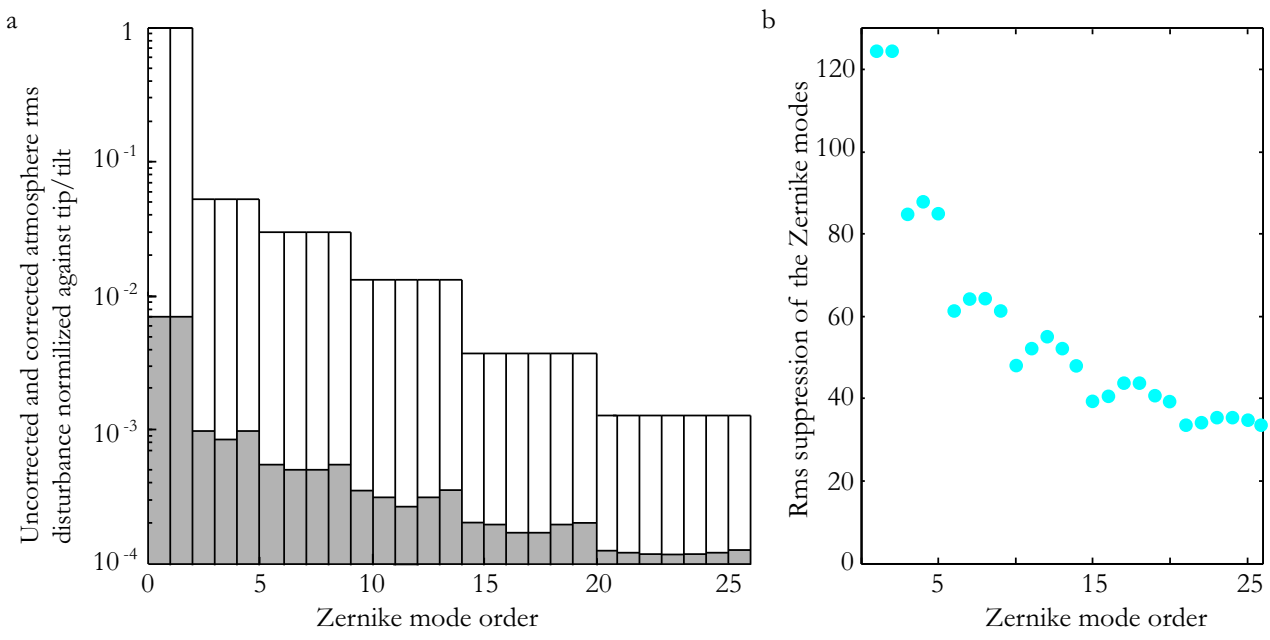

Figure 14. a) Contribution of each Zernike mode to the uncorrected and corrected atmospheric rms disturbance normalized with respect to the tip/tilt mode before correction; empty bars refer to the uncorrected, whereas filled bars refer to the corrected case. b) Suppression factors for the rms wavefront errors of the lowest 25 Zernike components of the atmospheric phase noise.

Assuming frozen turbulence at wind speed $V$, the spatial power spectrum can be related to the temporal power spectrum with temporal frequency $\nu=V f_{x}$ as:

$$
W(\nu)=\frac{1}{V} \int_{-\infty}^{+\infty} P\left(\frac{\nu}{V}, f_{y}\right) \mathrm{d} f_{y}
$$

Using Eq. 7 and 8, the temporal power spectrum of a specific Zernike at the deformable mirror is

$$
W_{\mathrm{in}}(\nu)=\frac{0.0299}{V_{\mathrm{DM}} r_{0, \mathrm{DM}}^{5 / 3}} \int_{-\infty}^{+\infty}\left(\left(\frac{\nu}{V_{\mathrm{DM}}}\right)^{2}+f_{y}^{2}\right)^{-11 / 6}\left|M\left(\frac{\nu}{V_{\mathrm{DM}}}, f_{y}\right)\right|^{2} \mathrm{~d} f_{y}
$$

where the wind speed and Fried's parameter have been scaled from the $30 \mathrm{~m}$ entrance pupil to the $1 \mathrm{~m}$ deformable secondary mirror as $r_{0, \mathrm{DM}}=\frac{1}{30} r_{0}$ and $V_{\mathrm{DM}}=\frac{1}{30} V$. Note that only the first layer of the atmosphere in Table 2 is considered here.

The residual spectrum for each Zernike polynomial is then

$$
W_{\text {out }}(\nu)=|H(\nu)|^{2} W_{\text {in }}(\nu)
$$

where $H(\nu)$ is the system rejection transfer function in Fig. 12 . The power spectra for the uncorrected and the corrected tilt mode of the atmosphere are shown in Fig. 13. The Zernike spectra for radial degree $n$ are characterized by a cutoff frequency [17]

$$
\nu_{\mathrm{c}}=0.3(n+1) \frac{V}{D}
$$

The areas under the power spectra in Fig. 13 give the variance of the phase, from which the contribution of each Zernike mode can be computed, shown in Fig. 14a. The root mean square (rms) of the wavefront for the first 25 Zernike modes is normalized with respect to the uncorrected tilt mode. The Zernike suppression

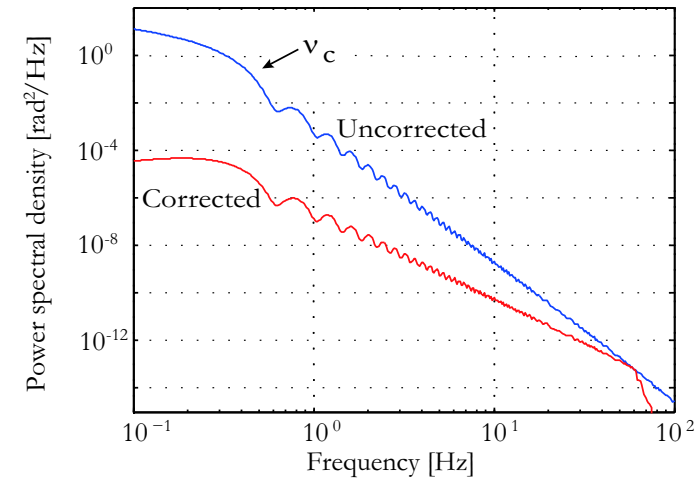

Figure 13. Temporal power spectra of the uncorrected and the corrected tilt term. (residual divided by input disturbance) is shown in Fig. 14b.

The mean square error for Kolmogorov turbulence is described by Noll [18] as $\sigma^{2}=1.0299\left(D / r_{o}\right)^{5 / 3}$. The mean-square residuals are suppressed by a factor of about 700 (root mean square reduction of 26). Thus, using Maréchal's approximation, the Strehl ratio is 0.8 for our case. 

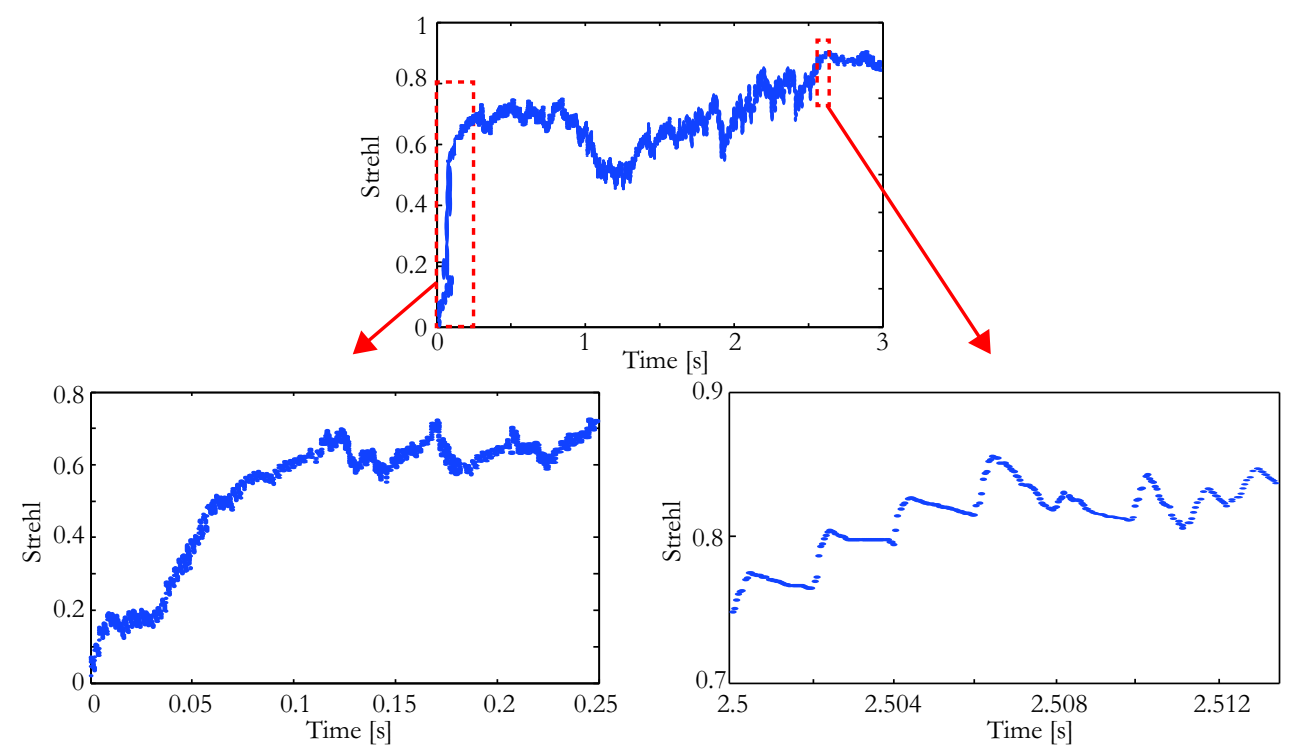

Figure 15. A three second time-domain simulation, where the Strehl ratio is computed for each time point. The zoom windows show the start-up transient of the system from its rest position and a representative operation time interval, respectively.

\subsection{Time Domain Simulation}

A time-domain simulation using the three-layer atmosphere in Table 2 also demonstrates feasibility. The layers obey Kolmogorov statistics given by Eq. 7, and frozen turbulence is again assumed.

The phase screen $\varphi(\mathbf{r})$ describes the phase difference added to a wavefront when passing through a thin turbulent layer. The phase screen is obtained by filtering a random function $J(\boldsymbol{f})$ with the square root of the power spectrum of the two-dimensional phase, $W_{\text {in }}(\boldsymbol{f})$,

$$
\varphi(\mathbf{r})=\mathcal{F}^{-1}\left(\sqrt{W_{\text {in }}(\boldsymbol{f})} J(\boldsymbol{f})\right)
$$

A fourth-order Runge-Kutta method is used with fixed integration interval of $50 \mu$ s. The sampling periods of the phase screens and the wavefront sensor are $1 \mathrm{~ms}$ and $2 \mathrm{~ms}$, respectively. Linear interpolation is used to update the phase screen during a sampling period.

A result from a three second simulation is shown in Fig. 15. The two zooms show the initial transient response of the mirror when it is commanded from its rest position, and a $14 \mathrm{~ms}$ window illustrating the typical behavior of the mirror during operation. The start-up transient has a duration of $0.1 \mathrm{~s}$ due to actuator saturation. Further, the effect of temporal WFS sampling is apparent in the right zoom of Fig. 15.

The Strehl ratio is computed using Maréchal's approximation for all nodes across the mirror. If the performance during the start-up transient is not considered, the average Strehl ratio is 0.77. If our DM is replaced by an ideal DM, the average Strehl ratio is increased to 0.81 for the same phase screen. In either case, the phase lag is the main factor limiting AO performance; the dynamic performance of the simulated DM with our proposed control algorithm and actuator and sensor dynamics does not significantly limit AO performance.

\section{CONCLUSIONS}

We have demonstrated feasibility of a control approach that has the potential to enable a low-cost concept for a large deformable mirror for adaptive optics. Use of voice-coil (force) actuators results in a lightly-damped mirror with many structural modes within the AO control bandwidth, resulting in the need for feedback from mechanical sensors to obtain dynamic response sufficient for AO. The control system must compensate for both resonant behaviour within the control bandwidth, and significant differences in the response between low and high spatial frequencies, while relying on inexpensive sensor/actuator hardware with associated constraints.

Inexpensive electromechanical sensors can be used because precision is not required quasi-statically, nor do the sensors need to be collocated with the actuators to provide active damping. The first of these follows because 
the AO system provides an "outer" wavefront-sensor based feedback loop that compensates for low-frequency DM errors. The non-collocation has been shown to be acceptable provided it is small enough that the resulting loss of positivity occurs above the acoustic limit of the mirror; this allows rate feedback to be stably implemented despite the resulting phase-lag.

In addition to active damping, the control also includes a local approach wherein a force pattern is applied to a family of nearby actuators in response to a displacement command at a single actuator. This contribution ensures that the mirror has similar dynamic behavior for all the spatial frequencies that can be introduced by the actuators. Because this relies only on local model information, it has the potential to be more robust than a global model-based solution. The local approach can be interpreted as compensating for crosstalk between neighbouring actuators.

The performance of the low-cost DM system has been simulated within an AO system using both an analytical Zernike polynomial expansion of the atmosphere and a time-domain simulation. These agree well, with the timedomain simulation giving an average Strehl ratio of 0.77, compared to a Strehl of 0.81 for an ideal DM. With low-cost sensors and actuators, the dynamic behavior of the deformable mirror is not the same as that of a typical DM, but this demonstrates that it still provides good performance within an adaptive optics system.

The control approach described here is also of potential interest for future space telescopes [19]. The ability to provide control despite many flexible modes within the bandwidth could be an enabler for very thin and hence lightweight mirrors.

\section{REFERENCES}

1. Crepy, B., Chaillot, S., Cola, M., Conan, J. M., Cousty, R., Dimmler, M., Dournaux, J. L., Zotti, S. D., Gabriel, E., Gasmi, R., Grasser, R., Hubin, N., Jagourel, P., Jochum, L., Locre, F., Madec, P.-Y., Morin, P., Mueller, M., Petit, G., Petitga, D., Roland, J. J., Sinquin, J. C., and Vernet, E., "Demonstration prototype and breadboards of the piezo stack M4 adaptive unit of the E-ELT," Proc. SPIE 7736, 2010.

2. Riccardi, A., Xompero, M., Zanotti, D., Busoni, L., Vecchio, C. D., Salinari, P., Ranfagni, P., Zappellini, G. B., Biasi, R., Andrighettoni, M., Gallieni, D., Anaclerio, E., Martin, H. M., and Miller, M., "The adaptive secondary mirror for Large Binocular Telescope: results of acceptance laboratory test," Proc., SPIE 5169, 2008.

3. Quiros-Pacheco, F., Busoni, L., Aqapito, G., Esposito, S., Pinna, E., Puqlisi, A., and Riccardi, A., "First light AO (FLAO) system for LBT: performance analysis and optimization," Proc., SPIE 77736, 2010.

4. MacMynowski, D. G., Heimsten, R., and Andersen, T., "Distributed force control of deformable mirrors," European J. Control, Vol. 17, No. 3, pp. 249-260, 2011.

5. Heimsten, R., Owner-Petersen, M., Ruppel, T., MacMynowski, D. G., and Andersen, T., "Suppressing low-order eigenmodes with local control for deformable mirrors," Optical Engineering, Vol. 51, 2012.

6. Heimsten, R., MacMynowski, D. G., Andersen, T., and Owner-Petersen, M., "Concept, modeling, and performance prediction of a low-cost deformable mirrors," Applied Optics, Vol. 51, No. 5, pp. 515-524, 2012.

7. Andersen, T., Garpinger, O., Owner-Petersen, M., Bjoorn, F., Svahn, R., and Ardeberg, A., "Novel concept for large deformable mirrors," Opt. Eng., Vol. 45, 2006.

8. Miller, D. W. and Grocott, S. C. O., "Robust Control of the Multiple Mirror Telescope Adaptive Secondary Mirror," Optical Engineering, Vol. 38, No. 8, pp. 1276-1287, August 1999.

9. Kulkarni, J., D'Andrea, R., and Brandl, B., "Application of distributed control techniques to the adaptive secondary mirror of Cornell's Large Atacama Telescope," Proc., SPIE 4839, 2003, pp. 750-756.

10. MacMartin, D. G., "Local, hierarchic, and iterative reconstructors for adaptive optics," J. of the Optical Society of America, A, Vol. 20, No. 6, pp. 1084-1093, 2003.

11. Andersen, T. and Enmark, A., Integrated Modeling of Telescopes, Springer, 2011.

12. Preumont, A., Vibration Control of Active Structures: An Introduction, Kluwer Academic Publishers, 2002.

13. MacMartin, D. G. and Hall, S. R., "Control of Uncertain Structures using an $H_{\infty}$ Power Flow Approach," AIAA J. Guidance Control and Dynamics, Vol. 14, No. 3, pp. 521-530, May-June 1991.

14. Benassi, L. and Elliott, S. J., "The equivalent impedance of power-minimising vibration controllers on plates," $J$. Sound and Vibration, Vol. 283, pp. 47-67, 2005.

15. Lyon, R. H., Machinery Noise and Diagnostics, Butterworth Publishing, June 1987.

16. Hardy, J. W., Adaptive Optics for Astronomical Telescopes, Oxford University Press, 1998. Oxford Series on Optical and Imaging Sciences.

17. Conan, J. M., Rousset, G., and Madec, P. Y., "Wave-front temporal spectra in high-resolution imaging through turbulence," J. Opt. Soc. Am. A, Vol. 12, 1995.

18. Noll, R. J., "Zernike Polynomials and Atmospheric Turbulence," J. Optical Society of America, Vol. 66, pp. 207-211, 1976.

19. MacMynowski, D. G., "Control of a hypersegmented space telescope," AIAA J. Guid. Control Dyn., Vol. 35, No. 3, 2012 . 